\title{
The p53 family members have distinct roles during mammalian embryonic development
}

\author{
Jeanine L Van Nostrand ${ }^{1}$, Margot E Bowen ${ }^{1}$, Hannes Vogel ${ }^{2}$, Maria Barna ${ }^{3,4}$ and Laura D Attardi, ${ }^{*, 1,4}$
}

The $p 53$ tumor suppressor is a member of a multi-protein family, including the p63 and p73 transcription factors. These proteins can bind to the same consensus sites in DNA and activate the same target genes, suggesting that there could be functional redundancy between them. Indeed, double mutant mice heterozygous for any two family member-encoding genes display enhanced cancer phenotypes relative to single heterozygous mutants. However, whether the family members play redundant roles during embryonic development has remained largely unexplored. Although $p 53^{-/-} ; p 73^{-1-}$ mice are born and manifest phenotypes characteristic of each of the single mutants, the consequences of combined deficiency of p63 and either p53 or p73 have not been elucidated. To examine the functional overlap of p53 family members during development, we bred and analyzed compound mutant embryo phenotypes. We discovered that double knockout embryos and five allele knockout embryos only displayed obvious defects accounted for by loss of single p53 family members. Surprisingly, at mid-gestation (E11), we identified a single viable triple knockout embryo that appeared grossly normal. Together, these results suggest that the p53 family is not absolutely required for early embryogenesis and that p53 family members are largely non-redundant during early development.

Cell Death and Differentiation (2017) 24, 575-579; doi:10.1038/cdd.2016.128; published online 17 February 2017

The p53 protein is a critical tumor suppressor, as indicated by the findings that $p 53$ is mutated in over half of all human tumors and that p53-null mice develop cancer with complete penetrance. ${ }^{1-4}$ p53 is a transcription factor, which, when activated in response to various cellular stress signals, induces expression of numerous downstream targets important for its cellular functions, including cell-cycle arrest and apoptosis, mechanisms to limit cellular proliferation. ${ }^{5}$ Although p53 knockout mice do not die until they succumb to tumors as adults, underscoring a predominant role for p53 in tumor suppression, ${ }^{2-4}$ more careful inspection of $p 53$-null embryos demonstrated that a subset display developmental phenotypes. ${ }^{6,7}$ In all, 8-16\% of p53-null embryos, depending on the genetic background, die perinatally with the cranial neural tube closure defect exencephaly, a phenotype observed mostly in females. ${ }^{6,7}$ p53-null embryos also manifest partially penetrant defects in other structures, such as eyes and teeth. ${ }^{7-9}$ This relatively minor role in development relative to tumor suppression contrasts with that of most tumor suppressor genes, whose knockout in mice typically provokes fully penetrant embryonic lethality. ${ }^{10}$

Subsequent to the discovery of p53, two related transcription factors-p63 and p73-were identified. ${ }^{11}$ The three proteins exhibit significant sequence similarity in their sequence-specific DNA-binding ( 63\%), oligomerization $(\sim 37 \%)$ and transactivation ( 22\%) domains and can bind to the same consensus sites in DNA and activate the same target genes. ${ }^{11}$ However, unlike $p 53$, with a paramount role in cancer suppression, p63 and p73 play more central developmental roles. p63-nullizygosity results in perinatal lethality accompanied by a failure of stratified epithelium and limb development, and by craniofacial and skeletal defects. ${ }^{12,13}$ p73-nullizygosity results in lethality within several weeks after birth, along with a host of phenotypes, including reduced size, hippocampal dysgenesis, hydrocephalus, chronic infections and inflammation, and pheromone-sensing defects that impede breeding. ${ }^{14}$ At least some of these phenotypes are attributable to defects in multiciliogenesis. ${ }^{15,16}$

Given the similarity to $p 53$, a role for p63 and p73 in tumor suppression has also been investigated. While not found to be commonly mutated in human cancer, p63- or p73-heterozygous mice on some genetic backgrounds display a slightly increased predisposition to certain tumor types relative to wildtype mice, typically associated with loss of heterozygosity $(\mathrm{LOH}),{ }^{17}$ and $E \mu-M y c$ mice heterozygous or null for $p 73$ present with higher rates of lymphoma dissemination than control mice. ${ }^{18}$ Moreover, compound mutant mice heterozygous for any two of the $p 53, p 63$, and $p 73$ loci exhibit reduced tumor latency, higher tumor burden and enhanced rates of metastasis relative to single heterozygotes. ${ }^{17,19}$ Finally, p73 loss enhances the tumor predisposition of $p 53$-null mice. ${ }^{19}$ These findings suggest that the family members play redundant roles in cancer suppression.

Although there is evidence for redundancy between p53 family members in suppressing cancer, it remains unclear how much the family members compensate for each other during

\footnotetext{
${ }^{1}$ Division of Radiation and Cancer Biology, Department of Radiation Oncology, Stanford University School of Medicine, Stanford, CA 94305, USA; ${ }^{2}$ Department of Pathology, Stanford University School of Medicine, Stanford, CA 94305, USA; ${ }^{3}$ Department of Developmental Biology, Stanford University School of Medicine, Stanford, CA 94305, USA and ${ }^{4}$ Department of Genetics, Stanford University School of Medicine, Stanford, CA 94305, USA

${ }^{*}$ Corresponding author: LD Attardi, Division of Radiation and Cancer Biology, Department of Radiation Oncology, Stanford University, CCSR-South, Room 1255, 269 Campus Drive, Stanford, CA 94305-5152, USA. Tel: +650 725 8424; Fax: +650 723 7382; E-mail: attardi@stanford.edu

Received 31.8.16; revised 4.10.16; accepted 4.10.16; Edited by G Melino; published online 17.2.2017
} 
embryonic development. The lethality observed in singlefamily member knockout mice typically occurs after birth, and it may be that there is not a more dramatic phenotype in these knockouts during development because of redundancy or compensation between family members. In terms of expression patterns, p53 mRNA is expressed ubiquitously during early mouse development, but then exhibits a more restricted pattern in differentiating organs later in development. ${ }^{20}$ p63 protein is expressed in the basal layer of various stratified epithelia, such as the epidermis, esophagus, bladder, and mammary gland, $^{12,13,21}$ whereas p73 RNA/protein is expressed primarily in the nervous system with some expression in testis germ cells and in specific epithelia such as the epidermis. ${ }^{14,22-24}$ Thus, these expression patterns suggest that the most likely tissues in which family members might play redundant functions would be epithelia such as the epidermis. Here, we address the redundancy between p53 family members during embryogenesis by intercrossing previously generated $p 53, p 63$, and $p 73$ heterozygous mutant mice and analyzing the resulting compound mutant mice at various time points throughout development. ${ }^{6,13,14}$ As previous studies have already reported that $p 53^{-/-} ; p 73^{-/-}$ compound male mice are born at Mendelian ratios, with compound mutant females displaying neonatal mortality due to the defects attributable to p53 loss, namely exencephaly, ${ }^{19,25}$ we have primarily analyzed the viability and developmental phenotypes of $p 53^{-1-} ; p 63^{-1-}$, and $p 63^{-1-} ; p 73^{-1-}$ mice..$^{6,13,14}$

Analysis of compound null progeny from $p 53^{+/-} ; p 63^{+/-}$ intercrosses revealed no viable mice at weaning, consistent with $p 63^{-1}$ embryos displaying perinatal lethality due to dehydration caused by defects in epidermal stratification. ${ }^{12,13}$ However, we observed near-expected Mendelian numbers of live $p 53^{-1} ; p 63^{-/}$embryos at both E10.5 and E16.5-E19.5, based on the presence of a heartbeat (Figure 1a). Wholemount and histological analyses of E10.5 and E16.5-E19.5 $p 53^{-/-} ; p 63^{-/-}$embryos revealed no obvious defects that would prevent late-stage viability (Figures $1 \mathrm{~b}$ and $2 \mathrm{a}$ ). Specifically, heart, liver, lung, intestine, and neuronal development appeared grossly normal. In contrast, the characteristic p63-null phenotypes of limb, epithelial, and craniofacial defects were equally apparent irrespective of p53 status. Moreover, the remnants of epidermis in $p 53^{-1} ; p 63^{-/}$mutants did not appear less intact than in $p 63^{-/}$embryos, as assessed by histology and staining for the basal cell marker K14 (Figure 2d). Thus, the combined loss of p53 and p63 in embryos does not appear to significantly compromise mouse development beyond simple p63 or p53 deficiency.

We next examined whether loss of both p63 and p73 has any effect on development. Interestingly, previous experiments examining $p 63^{-/} ; p 73^{-/}$MEFs suggested that combined p63 and p73-nullizygosity phenocopies p53 loss in apoptosis assays, both in the embryonic CNS and in oncogene-expressing fibroblasts. ${ }^{26}$ Upon intercrossing $p 63^{+/-}$; $p 73^{+/-}$mice, we observed no viable $p 63^{-1} ; p 73^{-/}$mice after birth, as expected from p63 deficiency. However, analyses of embryos late in embryogenesis at E16.5-E18.5 revealed viable $p 63^{-1} ; p 73^{-/}$embryos at normal Mendelian ratios (Figure 1c). Further analyses revealed limb, epithelial and craniofacial defects attributable to p63 loss (Figures 1d and 2b). However, there were no apparent additional defects due to the added loss of p73, as heart, liver, lung, intestine, and neuronal development appeared globally normal. Notably, we did not observe any dramatic brain phenotypes that are sometimes observed in $p 73^{-/}$mice, such as hydrocephalus, although this could be due to low penetrance ( 20\%) and/or genetic background. ${ }^{14,15}$ In addition, the residual epidermis did not show any enhanced defects compared to the single mutants (Figure 2d). Notably, compound p63 and p73 deficiency did not induce the exencephaly seen in some $p 53^{-1}$ embryos, indicating that combined loss of $p 63$ and $p 73$ does not recapitulate $p 53$ loss in the context of the neural tube. Thus, $p 63^{-1} ; p 73^{-/}$embryos showed no dramatic developmental defects beyond those observed in single knockout embryos.

Since double knockout of p53 family members did not significantly affect embryonic development, we sought to determine whether loss of any five alleles or all six alleles of the p53 family would result in any additional developmental defects. Given the poor odds of obtaining triple knockout embryos (1 in 64), we first examined the effects of loss of any five alleles of the p53 family on normal development (mice null for two genes and heterozygous for the remaining gene; 1 in 32 odds). We intercrossed $p 53^{+/-} ; p 63^{+/-} ; p 73^{+/-}$triple heterozygous mice and analyzed viability at mid-gestation. Analysis of embryos at both E10.5 and E13.5 revealed viable embryos of all possible combinations: $p 53^{-/-} ; p 63^{-1-} ; p 73^{+/-}$, $p 53^{-/-} ; p 63^{+/-} ; p 73^{-/-}$, and $p 53^{+/-} ; p 63^{-/-} ; p 73^{-/-}$, as determined by the presence of a heartbeat (Figure 1e). Wholemount and histological analyses revealed only defects attributable to p63-nullizygosity (for example, limb and craniofacial defects), and, in a subset of embryos, defects associated with p53-nullizygosity (e.g. exencephaly) (Figures 1f and 2c). Somite number and heart, liver, lung, and neuronal development appeared globally normal, suggesting that these embryos may also be viable at later stages of development. Thus, loss of five out of six alleles of the p53 family does not compromise development through mid-gestation.

Surprisingly, we also identified a single $p 53^{-1-} ; p 63^{-1-}$; $p 73^{-/}$embryo at E11.0, based on PCR analysis of both yolk sac and tail DNA (Figures 1e and g). This triple knockout embryo was viable, as determined by the presence of a heartbeat. Whole-mount analysis of the triple knockout embryo uncovered only p63-null associated defects similar to a $p 63^{-/}$littermate and not the p53-null associated defect exencephaly (Figure 1h). Although somite number, liver structure, and neural tube closure of this $p 53^{-1-} ; p 63^{-1-}$; $p 73^{-1}$ embryo looked developmentally typical, histological analyses of serial sections revealed hypomorphic cardiac cushion development relative to p63-null and other littermates (Figures $1 \mathrm{~h}$ and $2 \mathrm{e}$ ). Collectively, these findings indicate that the p53 family members are not strictly necessary for early development, but that they may play redundant roles in specific developmental processes. Given the low odds of obtaining embryos of the triple knockout genotype, it is also difficult to ascertain to what extent these embryos might be underrepresented. Attempts to increase the odds of obtaining triple knockout embryos by using homozygous null breeders were complicated by the early mortality of p63- and $p 73-$ null mice, the high incidence of early cancer development in 
$p 53-n u l l$ mice, and the limited ability of $p 73$ - and $p 53-$ null mice to breed due to the absence of pheromone receptors and reduced fertility, respectively. ${ }^{2-4,12-14,24,27}$ In future, it will be

a

\begin{tabular}{|lccc|}
\hline \multicolumn{4}{|c|}{$p 5^{+/-} ; p 63^{+/-} \times p 53^{+/-} ; p 63^{+/-}$} \\
\hline \multicolumn{4}{|c|}{ Observed Number (Expected) } \\
\cline { 2 - 4 } & Total & $\begin{array}{l}p 53_{-1-} \\
p 63-/-\end{array}$ \\
\hline E10.5 & 33 & $1(2.1)$ & $p=0.12$ \\
\hline E16.5-19.5 & 104 & $4(6.5)$ & $p=0.70$ \\
\hline
\end{tabular}

b

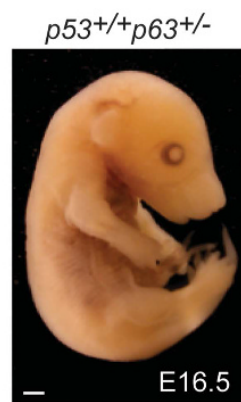

p53\%-p63\%-

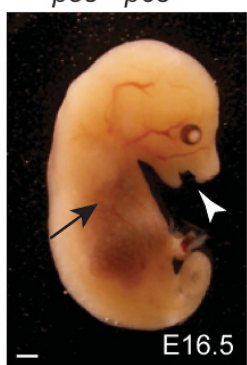

important to attempt to analyze a larger cohort of triple knockout embryos to fully understand the role of the p53 family in development. c

\begin{tabular}{|c|c|c|c|}
\hline \multicolumn{4}{|c|}{$p 63^{+/-} ; p 73^{+/-} \times p 63^{+/-} ; p 73^{+/-}$} \\
\hline \multicolumn{4}{|c|}{ Observed Number (Expected) } \\
\hline & Total & $\begin{array}{l}\text { p63 - } \\
\text { p73 - }\end{array}$ & \\
\hline E10.5 & 48 & $2(3)$ & $\mathrm{p}=0.04$ \\
\hline E13.5 & 54 & $6(3.4)$ & $p=0.01$ \\
\hline E16.5-18.5 & 143 & $10(8.9)$ & $p=0.42$ \\
\hline
\end{tabular}

d

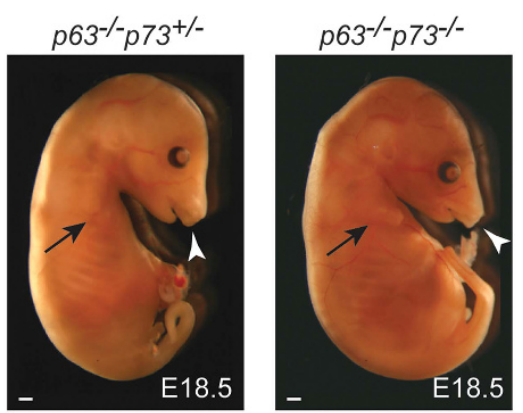

e

\begin{tabular}{|c|c|c|c|c|c|c|c|c|c|}
\hline \multicolumn{10}{|c|}{$p 53^{+/-} ; p 63^{+/-} ; p 73^{+/-} \times p 53^{+/-} ; p 63^{+/-} ; p 73^{+/-}$} \\
\hline & \multicolumn{8}{|c|}{$\begin{array}{c}\text { Genotype } \\
\text { Observed Number (Expected) }\end{array}$} & \\
\hline & & $p 53+/+$ & $+/-$ & $-1-$ & -1 & $-1-$ & -1 & $\%$ & \\
\hline & & p63 -/ & - & +/+ & $+/-$ & - & - & $\%$ & \\
\hline & Total & $p 73$ - & $-1-$ & -1 & - & $+/+$ & $+/-$ & - & \\
\hline E10.5-11.0 & 146 & $2(2.3)$ & $3(4.6)$ & $2(2.3)$ & $6(4.6)$ & $2(2.3)$ & $4(4.6)$ & $1(2.3)$ & $p=0.62$ \\
\hline E13.5 & 75 & $1(1.2)$ & $3(2.3)$ & $3(1.2)$ & $3(2.3)$ & $1(1.2)$ & $2(2.3)$ & $0(1.2)$ & $p=0.52$ \\
\hline
\end{tabular}

$\mathbf{f}$

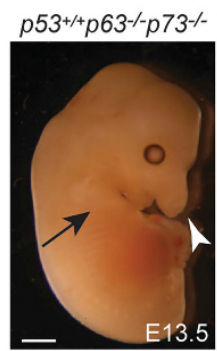

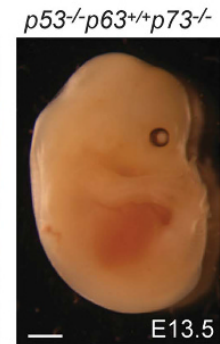

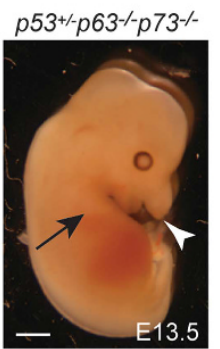

g

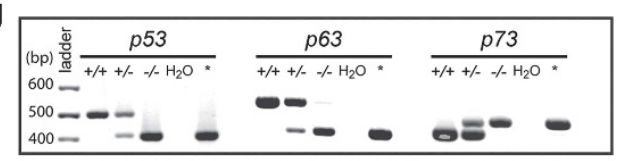

h

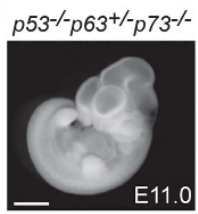

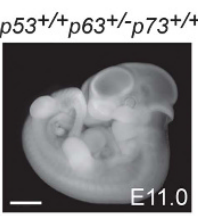

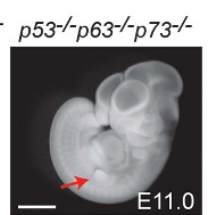

Figure 1 Viability of p53 family compound mutant embryos. (a) The observed numbers (and expected Mendelian numbers) of $p 53^{-/-} ; p 63^{-/}$embryos recovered at the indicated embryonic $(E)$ stages from intercrosses of $p 53^{+/-} ; p 63^{+/-}$mice. Genotypes were determined by PCR analysis of yolk sac DNA. Primer sequences are available upon request. The total number of conceptuses isolated is indicated. Significance as denoted by $P$ is determined by the $\chi^{2}$ test. (b) Whole-mount images of E16.5 $p 53^{-1-} ; p 63^{-/-}$ embryo (right) compared to control littermate (left), showing lack of limbs (arrow) and craniofacial defects (arrowhead). Scale bars indicate $1 \mathrm{mM}$. (c) The observed numbers (and expected Mendelian numbers) of $p 63^{-/} ; p 73^{--}$embryos recovered at the indicated embryonic (E) stages from intercrosses of $p 63^{+/-} ; p 73^{+/-}$mice. Genotypes were determined by PCR analysis of yolk sac DNA. The total number of conceptuses isolated is indicated. Significance as denoted by $P$ is determined by the $\chi^{2}$ test. (d) Whole-mount images of E18.5 $p 63^{-1-} ; 7^{-/-}$embryo (right) compared to control p63-null littermate (left), showing lack of limbs (arrow) and craniofacial defects (arrowhead). Scale bars indicate $1 \mathrm{mM}$. (e) The observed numbers (and expected Mendelian number) of compound mutant embryos recovered from p53;p63;p73-heterozygous intercrosses at the indicated embryonic (E) ages. Genotypes were determined by PCR analysis of yolk sac DNA. The total number of conceptuses isolated is indicated. Significance as denoted by $P$ is determined by $\chi^{2}$ test. Green: double knockout; Blue: five allele knockout; Red: triple knockout. (f) Whole-mount images of E13.5 $p 53^{+/-} ; p 63^{-/-} ; p 73^{-1-}$ embryo (right) compared to control littermates (left \& middle), showing lack of limbs (arrow) and craniofacial defects (arrowhead) in the p63-null embryos. Scale bars indicate $1 \mathrm{mM}$. (g) PCR analysis for $p 53, p 63$ and $p 73$ alleles in triple knockout embryo ( $\left.{ }^{*}\right)$ relative to wild-type, heterozygous, null, and negative $\left(\mathrm{H}_{2} \mathrm{O}\right)$ controls. DNA was derived from embryonic tail. (h) Whole-mount images of ethidium bromide-stained UV-imaged $p 53^{-1-} ; p 63^{-1} ; p 73^{-/-}$embryo (right) at E11.0 compared to control littermates (left). The $p 53^{-/} ; p 63^{-1-} ; p 73^{-/}$embryo has retarded limb development (arrow). Scale bars indicate 500 microns. Mice were maintained on a mixed 129/Sv;C57BL/6J background. Animal work was done according to the Stanford University APLAC 
a

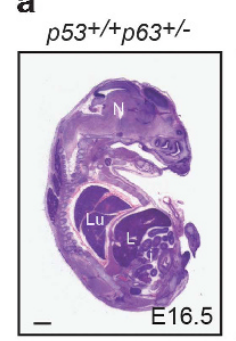

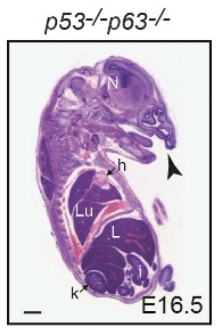

b

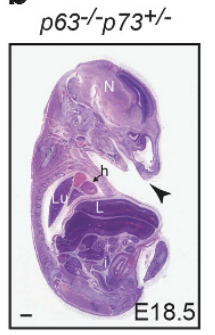

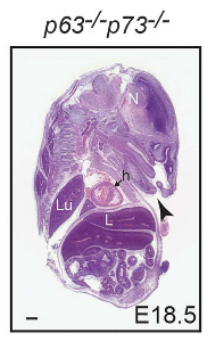
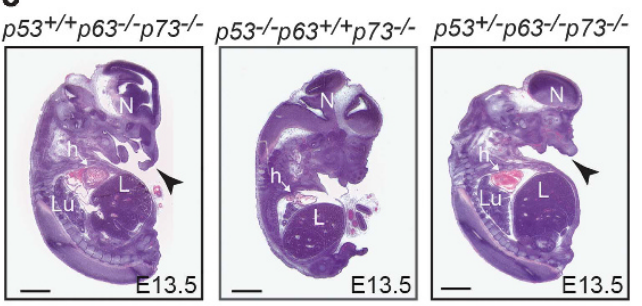

d
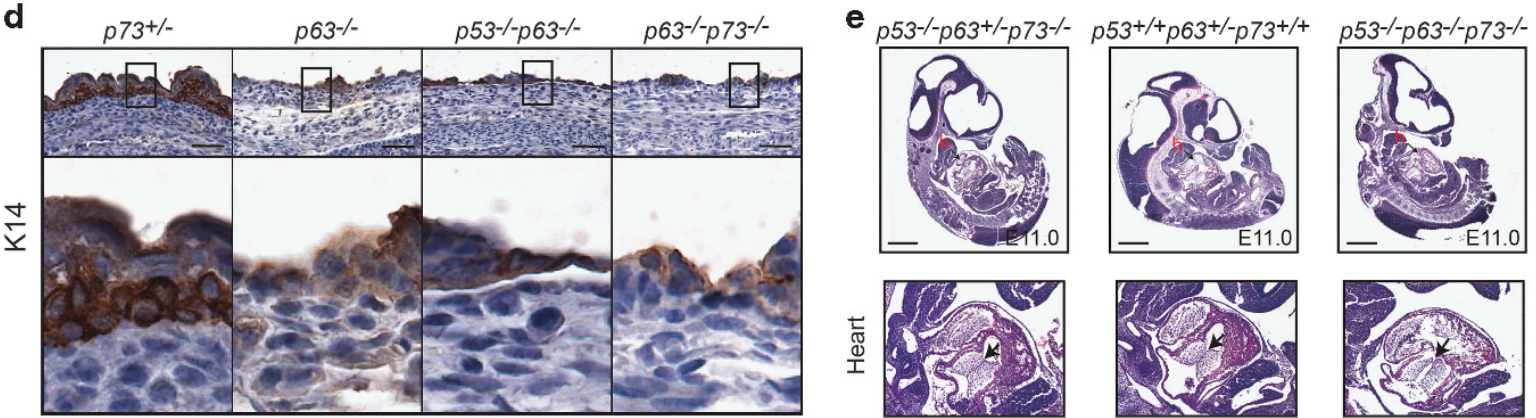

Figure 2 Histological analysis of p53 family compound mutant embryos. (a) Representative hematoxylin and eosin (H\&E)-stained images of sagittal sections of E16.5

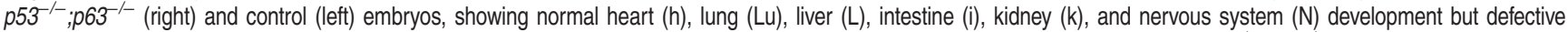
craniofacial development (arrowhead). Scale bars indicate $1 \mathrm{mM}$. (b) Representative H\&E-stained images of sagittal sections of E18.5 p63 ${ }^{-1-} ; p 73^{-/-}$(right) and control p63-null (left) embryos, showing normal heart (h), lung (Lu), liver (L), intestine (i), and nervous system (N) development but defective craniofacial development (arrowhead). Scale bars indicate $1 \mathrm{mM}$. (c) Representative H\&E-stained images of sagittal sections of E13.5 $p 53^{+/-} ; \mathrm{p} 63^{-/-} ; \mathrm{p} 73^{-/-}$and control embryo sections, showing normal heart (h), lung (Lu), liver (L), and nervous system (N) development but defective craniofacial development (arrowhead). Scale bars indicate $1 \mathrm{mM}$. (d) K14 immunohistochemical analysis of the surface epithelium of E16.5 control (left) or mutant embryos (right). Scale bars indicate 50 microns. Bottom: magnified image of boxed area. Immunohistochemistry was

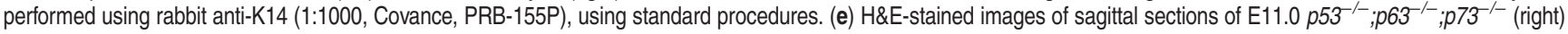
and control (left) embryos, showing normal liver and abnormal cardiac cushion development. Scale bars indicate 500 microns. Bottom: magnified image of cardiac cushion,

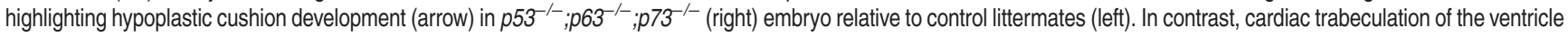
appeared normal

Of note, given that $\mathrm{p} 63$ and $\mathrm{p} 73$ exist in different isoformsthe TA isoforms with a complete transactivation domain and the $\triangle \mathrm{N}$ isoforms lacking the full TAD domain, and which typically inhibit the TA isoforms- it was formally possible that some phenotypes might have been rescued by compound deletion of multiple p53 family members. For example, if tissues where $\Delta \mathrm{Np} 63$ expression predominates are defective in p63-null animals because TAp73 is no longer inhibited, then the proper development of these tissues would be restored in $p 63^{-1-} ; p 73^{-\alpha}$ animals. However, in compound null embryos, we did not observe an amelioration of phenotypes typical of single mutants, suggesting that the lack of restraint of TA isoform function by $\Delta \mathrm{N}$ isoform expression does not underlie the observed phenotypes.

Given that the p53 family members are highly conserved, with homologous DNA-binding domains that allow them to bind the same consensus sites and regulate common target genes, it was expected that there would be functional redundancy between these proteins during development. While combined $p 53$ and p73 loss has previously been shown to be compatible with postnatal viability, ${ }^{19,25}$ we have now shown that loss of both p63 and p73 or p53 and p63 is compatible with viability until late gestation, with embryos manifesting only obvious defects characteristic of single mutants. The dispensability of the p53 family members for embryonic development is in stark contrast to the consequences of loss of other protein families during embryogenesis. For example, embryos with double knockout of members of the $\mathrm{Rb}$ family of cell-cycle regulators $(R b, p 107$, or $p 130$ ) consistently display earlier lethality than single mutants, underscoring the redundant functions of family members during embryogenesis. ${ }^{28-30}$ Furthermore, loss of all three $\mathrm{Rb}$ family members in the embryo elicits even more severe embryonic phenotypes. ${ }^{31}$ Thus, all Rb family members play redundant roles that can be revealed in any double mutant, and even more dramatically upon complete loss of the $\mathrm{Rb}$ family. These findings highlight the importance of redundancy between members of multi-protein families involved in cell-cycle regulation and apoptosis to ensure proper embryonic development and underscore the surprising lack of earlier lethality or significantly exacerbated developmental defects in the compound p53 family member mutant embryos.

The surprising lack of clear redundancy between the p53 family members during embryogenesis could relate to lack of either overlapping expression or compensatory upregulation of specific family members when other family members are ablated. Notably, we cannot completely rule out potential redundancy between family members very late in embryogenesis or more subtle redundant roles in the development of specific organs such as the heart. Interestingly, the lack of redundancy we observe contrasts with the redundancy observed between p53 family members in tumor studies, where more severe cancer phenotypes are observed with 
deficiency of multiple family members. ${ }^{17,19}$ These findings suggest the importance of cooperative action of the p53 family in certain physiological contexts such as cancer suppression, but not during early embryogenesis. Future investigation will better elucidate the interplay between p53 family members in later development and tumor suppression.

\section{NOTE}

While this manuscript was in press, Wang et al. reported using blastocyst injection of triple knockout (TKO) mouse embryonic stem cells, generated by CRISPR/Cas9-mediated inactivation of p53, p63 and p73, into wild-type embryos to analyze the phenotype of TKO embryos (Cell Stem Cell 20, 70-86, 2017). They showed that chimeric embryos displayed severe morphological defects starting at $\sim$ E7.5 and that the p53 family is essential for mesoendodermal differentiation. In contrast, using classical breeding strategies, we were able to identify one $\mathrm{E} 11.0 p 53^{-/-} ; p 63^{-/-} ; p 73^{-/-}$embryo that appeared normal except for heart endocardial cushion defects. Our result does not exclude a phenotype in some TKO embryos given the limited number of embryos we were able to obtain by breeding, but it does indicate that a relatively normal TKO embryo can develop. The difference in our results may relate to genetic background or potentially to the additional time required for compensatory pathways to be upregulated in our system.

\section{Conflict of Interest}

The authors declare no conflict of interest.

Acknowledgements. We thank $\mathrm{CY}$ Lin and CJ Lin for assistance with histological analyses and J Baker for helpful discussions. This work was supported by funding from the NSF and NCl (Grant Number 1F31CA167917-01) to JLVN, the Jane Coffin Childs Memorial Fund for Medical Research to MEB, and by funding from the ACS, LLS, and NIH (RO1 CA140875 and R35 CA197591) to LDA.

1. Varley JM. Germline TP53 mutations and Li-Fraumeni syndrome. Hum Mutat 2003; 21 : 313-320.

2. Donehower LA, Harvey M, Slagle BL, McArthur MJ, Montgomery CA Jr., Butel JS et al. Mice deficient for p53 are developmentally normal but susceptible to spontaneous tumours. Nature 1992; 356: 215-221.

3. Jacks T, Remington L, Williams BO, Schmitt EM, Halachmi S, Bronson RT et al. Tumor spectrum analysis in p53-mutant mice. Curr Biol 1994; 4: 1-7.

4. Purdie C, Harrison D, Peter A, Dobbie L, White S, Howie S et al. Tumour incidence, spectrum and ploidy in mice with a large deletion in the p53 gene. Oncogene 1994; 9: 603-609.

5. Bieging KT, Mello SS, Attardi LD. Unravelling mechanisms of p53-mediated tumour suppression. Nat Rev 2014; 14: 359-370.

6. Sah VP, Attardi LD, Mulligan GJ, Williams BO, Bronson RT, Jacks T. A subset of p53-deficient embryos exhibit exencephaly. Nat Genet 1995; 10: 175-180.

7. Armstrong JF, Kaufman MH, Harrison DJ, Clarke AR. High-frequency developmental abnormalities in p53-deficient mice. Curr Biol 1995; 5: 931-936.
8. Kaufman MH, Kaufman DB, Brune RM, Stark M, Armstrong JF, Clarke AR. Analysis of fused maxillary incisor dentition in p53-deficient exencephalic mice. J Anat 1997; 191(Pt 1): 57-64.

9. Rinon A, Molchadsky A, Nathan E, Yovel G, Rotter V, Sarig R et al. p53 coordinates cranial neural crest cell growth and epithelial-mesenchymal transition/delamination processes. Development 2011; 138: 1827-1838.

10. Jacks T. Tumor suppressor gene mutations in mice. Annu Rev Genet 1996; 30: 603-636.

11. Levrero M, De Laurenzi V, Costanzo A, Gong J, Wang JY, Melino G. The p53/p63/p73 family of transcription factors: overlapping and distinct functions. J Cell Sci 2000; 113: 1661-1670.

12. Mills AA, Zheng B, Wang X-J, Vogel H, Roop DR, Bradley A. p63 is a p53 homologue required for limb and epidermal morphogenesis. Nature 1999; 398: 708-713.

13. Yang A, Schweitzer R, Sun D, Kaghad M, Walker N, Bronson RT et al. p63 is essential for regenerative proliferation in limb, craniofacial and epithelial development. Nature 1999; 398: 714-718.

14. Yang A, Walker N, Bronson R, Kaghad M, Oosterwegel M, Bonnin J et al. p73-deficient mice have neurological, pheromonal and inflammatory defects but lack spontaneous tumours. Nature 2000; 404: 99-103.

15. Nemajerova A, Kramer D, Siller SS, Herr C, Shomroni O, Pena T et al. TAp73 is a central transcriptional regulator of airway multiciliogenesis. Genes Dev 2016; 30: $1300-1312$.

16. Marshall CB, Mays DJ, Beeler JS, Rosenbluth JM, Boyd KL, Guasch GLS et al. p73 is required for multiciliogenesis and regulates the Foxj1-associated gene network. Cell Rep 2016; 14: 2289-2300.

17. Flores ER, Sengupta S, Miller JB, Newman JJ, Bronson R, Crowley D et al. Tumor predisposition in mice mutant for p63 and p73: Evidence for broader tumor suppressor functions for the p53 family. Cancer Cell 2005; 7: 363-373.

18. Nemajerova A, Petrenko O, Trümper L, Palacios G, Moll UM Loss of p73 promotes dissemination of Myc-induced B cell lymphomas in mice. J Clin Invest 2010; 120 2070-2080.

19. Nemajerova A, Palacios G, Nowak NJ, Matsui S-i, Petrenko O. Targeted deletion of p73 in mice reveals its role in T cell development and lymphomagenesis. PLOS ONE 2009; 4 : e7784.

20. Schmid $\mathrm{P}$, Lorenz A, Hameister $\mathrm{H}$, Montenarh $\mathrm{M}$. Expression of $\mathrm{p} 53$ during mouse embryogenesis. Development 1991; 113: 857-865.

21. Pignon J-C, Grisanzio C, Geng Y, Song J, Shivdasani RA, Signoretti S. p63-expressing cells are the stem cells of developing prostate, bladder, and colorectal epithelia. Proc the Nat Acad Sci 2013; 110: 8105-8110.

22. Kamiya M, Takeuchi Y, Katho M, Yokoo H, Sasaki A, Nakazato Y. Expression of p73 in normal skin and proliferative skin lesions. Pathol Int 2004; 54: 890-895.

23. Hernández-Acosta NC, Cabrera-Socorro A, Morlans MP, Delgado FJG, Suárez-Solá ML, Sottocornola $R$ et al. Dynamic expression of the p53 family members p63 and p73 in the mouse and human telencephalon during development and in adulthood. Brain Res 2011; 1372: 29-40.

24. Holembowski L, Kramer D, Riedel D, Sordella R, Nemajerova A, Dobbelstein M et al. TAp73 is essential for germ cell adhesion and maturation in testis. J Cell Biol 2014; 204: 1173-1190.

25. Lee AF, Ho DK, Zanassi P, Walsh GS, Kaplan DR, Miller FD. Evidence that $\Delta$ Np73 promotes neuronal survival by p53-dependent and p53-independent mechanisms. J Neurosci 2004; 24: 9174-9184

26. Flores ER, Tsai KY, Crowley D, Sengupta S, Yang A, McKeon F et al. p63 and p73 are required for p53-dependent apoptosis in response to DNA damage. Nature 2002; 416: 560-564.

27. Hu W, Feng Z, Teresky AK, Levine AJ. p53 regulates maternal reproduction through LIF. Nature 2007; 450: 721-724.

28. Cobrinik D, Lee MH, Hannon G, Mulligan G, Bronson RT, Dyson N et al. Shared role of the pRB-related p130 and p107 proteins in limb development. Genes Dev 1996; 10 : 1633-1644.

29. Lee MH, Williams BO, Mulligan G, Mukai S, Bronson RT, Dyson N et al. Targeted disruption of p107: functional overlap between p107 and Rb. Genes Dev 1996; 10: 1621-1632.

30. Berman SD, West JC, Danielian PS, Caron AM, Stone JR, Lees JA. Mutation of p107 exacerbates the consequences of Rb loss in embryonic tissues and causes cardiac and blood vessel defects. Proc Natl Acad Sci USA 2009; 106: 14932-14936.

31. Wirt SE, Adler AS, Gebala V, Weimann JM, Schaffer BE, Saddic LA et al. G1 arrest and differentiation can occur independently of Rb family function. J Cell Biol 2010; 191 : 809-825. 